\title{
Revascularização cirúrgica do miocárdio sem utílização do circuito extracorpóreo em pacientes com infarto agudo do miocárdio tratados previamente com estreptoquinase intravenosa
}

Hermínio VEGA*, Luiz Eduardo V. LEĀO*, José Honório de Almeida Palma da FONSECA*, Walter José GOMES*, Lélio Alves SILVA**, João Nelson Rodrigues BRANCO^, Miguel Angel MALUF*, Expedito E. RIBEIRO**, Énio BUFFOLO*.

\section{RBCCV 44205-145}

VEGA, H.; LEÄO, L. E. V.; FONSECA, J. H. A. P.; GOMES, W. J.; SILVA, L.A. ; BRANCO, J. N. R.; MALUF, M. A.; RIBEIRO, E. E.; BUFFOLO, E. - Revascularizaçāo cirúrgica do miocárdio sem utilização do circuito extracorpóreo em pacientes com infarto agudo do miocárdio tratados previamente com estreptoquinase intravenosa. Rev. Bras. Cir. Cardiovasc., 6(3):153-157, 1991.

RESUMO: A utilização de agentes trombolíticos nas primeiras horas do infarto agudo do miocárdio tem sido aceita como um dos principais métodos de limitar-se a extensão do infarto. Entretanto, a persistência de estenose residual necessita de um método complementar de revascularização. Em nosso Serviço, temos realizado a revascularizaçäo cirurgica do miocárdio sem a utilizaçäo do circuito extracorpóreo de modo eletivo, com bons resultados. Utilizamos o método em 25 pacientes com diagnóstico de infarto agudo do miocárdio tratados dentro de seis horas do início dos sintomas com estreptoquinase intravenosa e um a 21 dias após (média de oito dias) a revascularizaçāo cirúrgica sem a utilizaçāo do circuito extracorpóreo. A média de idade do grupo foi de 53,8 anos, sendo a média da fração de ejeção medida pelo método de Dodge de $60 \%$; dez pacientees eram uniarteriais, 14 biarteriais e um triarterial, em 15 pacientes, o infarto localizava-se em parede anterior e em dez em parede posterior. Oito pacientes receberam uma ponte e 17, duas pontes. Em 15 casos utilizou-se a artéria torácica interna. A mortalidade hospitalar foi $0 \%$ e em nenhum caso houve necessidade de reoperaçāo por sangramento. A revascularizaçäo cirúrgica do miocárdio sem a utilização do circuito extracorpóreo é, pois, uma opçäo tática que pode ser utilizada em alguns pacientes com esse tipo de doença, com excelentes resultados em termos de morbidade e mortalidade hospitalares.

DESCRITORES: miocárdio, revascularizaçāo, cirurgia; miocárdio, infarto agudo, cirurgia; estreptoquinase.

\section{INTRODUÇĀO}

As tentativas mais modernas de limitar-se a extensão do infarto agudo do miocárdio são baseados no conceito de que a lesāo isquêmica não é homogênea em uma região do miocárdio nutrida por uma artéria coronária obstruída. De fato, nessa zona existe uma parte do miocárdio que irá evoluir para necrose independente da restauração do fluxo coronário, mas existe também uma zona isquêmica circunvizinha, que é potencialmente viável se o fluxo for restabelecido precodemente $4,7,10$.

O restabelecimento do fluxo irá, então, salvar a zona isquêmica que estaria destinada a lesão irreversível.

Trabalho realizado na Escola Paulista de Medicina. Disciplina de Cirurgia Cardiovascular. São Paulo. SP, Brasil.

Apresentado ao $18^{\circ}$ Congresso Nacional de Cirurgia Cardíaca. Rio de Janeiro, 5 e 6 de abril, 1991.

* Da Escola Paulista de Medicina.

** Do Hospital Unicór.

Endereço para separatas: Hermínio Vega. Hospital São Paulo. Cirurgia Cardiovascular. Rua Napoleão de Barros, 715.04024 Vila Clementino. São Paulo, SP, Brasil. 
VEGA, H.; LEẢO, L. E. V.; FONSECA, J. H. A. P.; GOMES, W. J.; SILVA, L.A. ; BRANCO, J. N. R.; MALUF, M. A.; RIBEIRO, E. E.; BUFFOLO, E. - Revascularizaçāo cirúrgica do miocárdio sem utilizaçäo do circuito extracorpóreo em pacientes com infarto agudo do miocárdio tratados previamente com estreptoquinase intravenosa. Rev. Bras. Cir. Cardiovasc., 6(3):153$157,1991$.

A associação de estreptoquinase intravenosa com revascularização cirúrgica do miocárdio tem sido utilizada por alguns autores no tratamento do infarto agudo do miocárdio ${ }^{2,6,8,9}$.

0 método apresentaria, como principais vantagens, o fato de poder ser iniciado precocemente na evolução do infarto agudo do miocárdio e atuar na estenose subjacente, promovendo a revascularização completa do miocárdio.

Temos realizado, em nosso Serviço, a revascularização cirúrgica do miocárdio sem a utilização do circuito extracorpóreo, de modo eletivo, com bons resultados ${ }^{\text {. }}$

Utilizamos, então, o método em 25 pacientes com diagnóstico de infarto agudo do miocárdio tratados dentro de seis horas do início dos sintomas com estreptoquinase intravenosa e um a 21 dias após (média de oito dias) a revascularização cirúrgica do miocárdio sem a utilização do circuito extracorpóreo.

Nossos objetivos são os de oferecer uma opção tática nesse tipo de paciente, bem como analisar nossos resultados em termos de mortalidade e morbidade pós-operatória.

\section{CASUÍSTICA E MÉTODOS}

Constituem objeto deste trabalho 25 pacientes com diagnóstico de infarto agudo do miocárdio e que foram submetidos, dentro de seis horas após o início dos sintomas, ao tratamento com estreptoquinase intravenosa e um a 21 dias após a revascularização cirúrgica do miocárdio sem a utilização do circuito extracorpóreo.

A média de idade do grupo era de 53,8 anos, sendo 22 pacientes do sexo masculino e três do feminino. Em 15 pacientes o infarto localizava-se na parede anterior e em dez na parede posterior.

Os critérios adotados para o diagnóstico de infarto agudo do miocárdio foram: dor precordial persistente, elevação do segmento ST de $2 \mathrm{~mm}$ ou mais em duas ou mais derivaçōes eletrocardiográficas, elevação da fração de MB de creatininoquinase, verificação em posterior estudo angiográfico das lesōes abstrutivas coronarianas e das alteraçōes da função contrátil do ventrículo esquerdo.

Nosso protocolo do tratamento do infarto agudo do miocárdio constituiu-se de orientação e consentimento do paciente, registro do eletrocardiograma e colheita de exames saguíneos, especialmente a fração $M B$ do enzima creatininoquinase, aplicação de 1.200 .000 unidades de estreptoquinase pela via intravenosa, divididos em duas doses de $600.000 \mathrm{U}$ com 15 minutos de intervalo, heparinização i.v., controlando-se a dose através de medidas do tempo parcial de tromboplastina (mantendo-a duas a três vezes o valor inicial), realização de estudo angiográfico aproximadamente dois dias após a infusão de estreptoquinase com medida da fração de ejeção do ventrículo esquerdo, de acordo com o método de Dodge ${ }^{3}$, sendo a média para o grupo todo de $60 \%$.

Nossos critérios adotados para constatar-se recanalização foram os seguintes: melhora ou desaparecimento da dor, tendência a normalização do segmento ST, aparecimento de arritmia de reperfusão, principalmente a taquicardia ventricular lenta, precocidade do pico de (KMB), vaso comprometido pérvio observado no estudo angiográfico. Seguindo esses critérios, 24 pacientes foram considerados recanalizados e somente em um o procedimento inicial não foi considerado bem sucedido. Foram selecionados para o procedimento cirúrgico somente pacientes portadores de lesōes na artéria interventrícular anterior, na artéria diagonal ou na artéria coronária direita. As lesōes na artéria circunflexa não podem ser abordadas através de esternotomia mediana longitudinal sem o levantamento da ponta do coração, tornando obrigatório o uso do circuito extracorpóreo parcial ou total. Em todos os 25 pacientes, - conjunto coração-pulmão artificial permaneceu montado de modo a ser rapidamente utilizado caso houvesse necessidade.

Ao mesmo tempo em que se procedeu à esternotomia, retirou-se a veia safena magna, de comprimento variável, preferencialmente do membro inferior direito, começando sua disecção ao nível da prega inguinal.

A artéria torácica interna foi, ou não, dissecada, levando-se em consideração a idade do paciente e a artéria coronária comprometida; tendo em vista esses critérios essa artéria foi utilizada em 15 pacientes.

Identificaram-se, então, as coronárias a serem tratadas, realizando-se a dissecção das mesmas nos locais a serem realizadas as anastomoses.

Terminado esse tempo preliminar, administrouse heparina $200 \mathrm{u} / \mathrm{kg}$ de peso e, a seguir, $5 \mathrm{mg}$ de verapamil, com o objetivo de baixar a pressão arterial sistêmica, diminuir o consumo de oxigênio do miocárdio e prevenir arritmias. Fios de Prolene 50 circundaram a artéria coronária em posição imediatamente proximal e distal ao local da anastomose.

A tração desses fios determinou a interrupção do fluxo coronário no segmento a ser abordado; aberta a artéria coronária e anastomose com sutura contínua tipo Blalock, com Prolene 7-0. Pinçamento lateral da aorta ascendente, seguido de incisão de forma circular e sutura proximal contínua com Prolene 6-0. 
VEGA, H.; LEẢO, L. E. V.; FONSECA, J. H. A. P.; GOMES, W. J.; SILVA, L.A. ; BRANCO, J. N. R.; MALUF, M. A.; RIBEIRO, E. E.; BUFFOLO, E. - Revascularizaçāo cirúrgica do miocárdio sem utilizaçāo do circuito extracorpóreo em pacientes com infarto agudo do miocárdio tratados previamente com estreptoquinase intravenosa. Rev. Bras. Cir. Cardiovasc., 6(3):153$157,1991$.

Realizada a anastomose, procedeu-se à revisão da hemostasia e neutralização de heparina com protamina (1:1).

Drenados o mediastino e a cavidade pleural, caso a mesma estivesse aberta com dreno plástico tubular sob-água. Fechada a toracotomia de madeira convencional. Terminado o ato cirúrgico, os pacientes foram encaminhados à Unidade de Recuperação Pós-Operatória, e mantidos com respiração controlada.

Foi realizado seguimento pós-operatório através de consultas ambulatoriais e/ou contato telefônico com todos os pacientes.

Foram analisados os resultados tardios para o grupo de 25 pacientes através da curva de Kaplan - Meier 5 .

\section{RESULTADOS}

Mortalidade imediata ou hospitalar, que compreende o óbito do paciente no pós-operatório imediato ou até 30 dias após a cirurgia, foi de $0 \%(0 /$ 25). Em termos de morbidade pós-operatória e levandose em conta apenas as complicaçōes hemorrágicas em vista do próprio tema do nosso trabalho, não houve a necessidade de nenhuma reoperação por sangramento no nosso grupo de pacientes.

A análise da curva de mortalidade (Gráfico 1) pelo método de Kaplan-Meier demonstra a ocorrência de óbitos tardios, sendo dois por infarto agudo do miocárdio, 24 e 42 meses após a cirurgia, respectivamente, e um por acidente vascular cerebral, um mês após a cirurgia. A sobrevida após dez

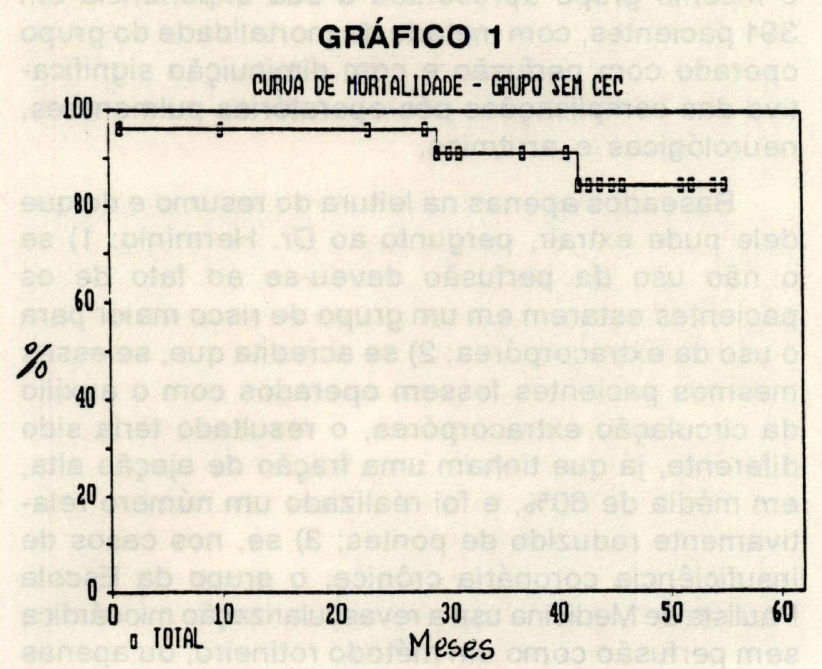

meses foi de $96 \%$, e, após 55 meses, de 83\%.

A análise da curva de morbidade tardia (Gráfico 2) pelo método de Kaplan-Meier, levando-se em consideração os parâmetros insuficiência cardíaca e angina, demonstra a ocorrência desse evento em três pacientes com uma probabilidade de ficar livre dessas complicaçōes após 23 meses, de 100\%, e após 46 meses, de $95 \%$.

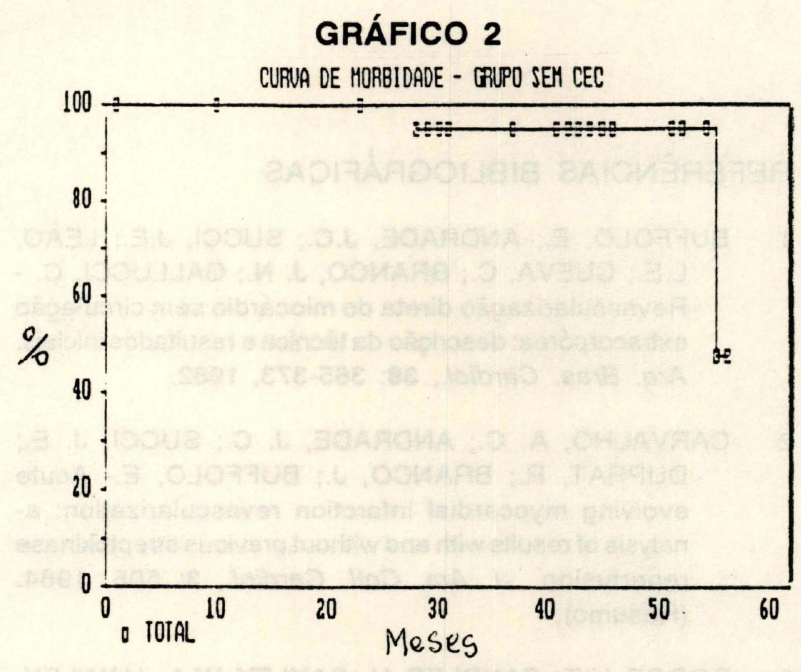

\section{DISCUSSĀO}

Há cerca de dez anos, temos realizado revascularização cirúrgica do miocárdio sem a utilização do circuito extracorpóreo. A experiência e segurança adquiridas durante o decorrer desses anos levou-nos a aplicar o procedimento em pacientes com infarto agudo do miocárdio tratados, inicialmente, com estreptoquinase intravenosa. Os resultados imediatos obtidos confirmam a validade do método particularmente em pacientes em que se faz necessária a realização de uma ou duas pontes em territórios da artéria coronária intrerventricular anterior e coronária direita. Acreditamos, também, que o método é valioso a fim de diminuir a morbidade hospitalar, uma vez que não causa as complicaçōes inerentes à circulação extracorpórea. Os bons resultados conseguidos em termos de morbidade tardia atestam que uma anastomose com boa qualidade pode ser obtida com este método.

Concluímos, pois, que o método é uma opção tática que pode ser utilizado em alguns pacientes com esse tipo de doença, com excelentes resultados em termos de morbidade e mortalidade hospitalar e tardia. 
VEGA, H.; LEĀO, L. E. V.; FONSECA, J. H. A. P.; GOMES, W. J.; SILVA, L.A. ; BRANCO, J. N. R.; MALUF, M. A.; RIBEIRO, E. E.; BUFFOLO, E. - Revascularização cirúrgica do miocárdio sem utilização do circuito extracorpóreo em pacientes com infarto agudo do miocárdio tratados previamente com estreptoquinase intravenosa. Rev. Bras. Cir. Cardiovasc., 6(3):153157, 1991.

\section{RBCCV 44205-145}

VEGA, H.; LEÄO, L. E. V.; FONSECA, J. H. A. P.; GOMES, W. J.; SILVA, L. A.; BRANCO, J. N. R.; MALUF, M. A.; RIBEIRO, E. E.; BUFFOLO, E. - Coronary artery bypass grafting without cardiopulmonary bypass in patients with myocardial infarction innitially treated with intravenous streptokinase. Rev. Bras. Cir. Cradiovasc. 6(3):153-157, 1991.

ABSTRACT: The authors have performed coronary artery bypass grafting without cardiopulmonary bypass in 25 patients with myocardial infarction treated innitially with intravenous streptokinase. Our goal is to offer a tatic option to this kind of patients. Our satisfactory results authorize us to continue to perform this procedure, particulary in patients who need one or two bypass graftings.

DESCRIPTORS: myocardial revascularization, surgery; myocardial infarct, surgery; streptokinase.

\section{REFERÊNCIAS BIBLIOGRÁFICAS}

1 BUFFOLO, E.; ANDRADE, J.C.; SUCCI, J.E.; LEĀO, L.E.; CUEVA, C.; BRANCO, J. N.; GALLUCCI, C. Revascularizaçäo direta do miocárdio sem circulação extracorpórea: descriçäo da técnica e resultados iniciais. Arq. Bras. Cardiol., 38: 365-373, 1982.

CARVALHO, A. C.; ANDRADE, J. C.; SUCCI, J. E.; DUPRAT, R.; BRANCO, J.; BUFFOLO, E.- Acute evolving myocardial infarction revascularization: analysis of results with and without previous streptokinase reperfusion. J. Am. Coll. Cardiol., 3: 505, 1984. (Resumo)

DODGE, H.T.; SANDLER, H.; BAXLEY, W.A.; HAWLEY, R. R. - Usefulliness and limitations of radiographic methods for determining left ventricular volume. Am. J. Cardiol., 18: 10-14, 1966.

JENNING, R. B. \& REIMER, K. A. - Factors involved in salvaging ischemic myocardium: effect of reperfusion of arterial blood. Circulation, 68: 25-36, 1983.

LEE, E. T. - Statistical methods for survival data analysis. New York, Lifetime Learning, 1980.

LOSMAN, J. G; FINCHUM, R. N.; NAPLE, D.; DACUMOS, G.; JANES, C.; WIFENSKY, A. S.; MARTIN, R. G.; BAILEY, M. T.; KAHN, D. - Myocardial surgical revascularization after streptokinase for acute myocardial infarction. J. Thorac. Cardiovasc. Surg., 89: 25-34, 1985.

REIMER, K. A.; LOWE, J. E.; RASMUSSEN, M. M. The wave-front phenomenon of ischemic death: myocardial infarct size vs duration of coronary occlusion in dogs. Circulation, 56: 786, 1977. (Resumo).

RENTROP, P.; BLANKE, H.; KARSECH, K. R.; KAISER, H.; KASTERING, H.; LEITZ, K. - Selective intracoronary thrombolysis in acute myocardial infarction and unstable angina pectoris. Circulation, 63: 307316, 1981.

VEGA, H. - Resultados imediatos e tardios da revascularização cirúrgica do miocárdio pós-trombólise coronariana com estreptoquinase intravenosa no tratamento do infarto agudo do miocárdio. São Paulo, 1990. (Tese - Mestrado. Escola Paulista de Medicina).

10 WETSTEIN, L.; SIMSON, M. B.; FELDMAN, P. O. Pharmacologic modification of myocardial ischemic. Circulation, 66: 548-554, 1982.

\section{Discussão}

\section{DR. ANTÔNIO PENNA}

Marília, $S P$

Todos nós temos acompanhado, desde 1981, os esforços da Escola Paulista de Medicina para implantar e divulgar o uso da revascularizaçāo do miocárdio sem o auxílio da circulaçāo extracorpórea.

Já em 1986, durante o nosso $13^{\circ}$ Congresso, o mesmo grupo apresentou a sua experiência em 391 pacientes, com metade da mortalidade do grupo operado com perfusão e com diminuição significativa das complicaçōes pós-operatórias pulmonares, neurológicas e arritmias.

Baseados apenas na leitura do resumo e do que dele pude extrair, pergunto ao Dr. Hermínio: 1) se o não uso da perfusão deveu-se ao fato de os pacientes estarem em um grupo de risco maior para o uso da extracorpórea; 2) se acredita que, se esses mesmos pacientes fossem operados com o auxílio da circulação extracorpórea, o resultado teria sido diferente, já que tinham uma fração de ejeção alta, em média de $60 \%$, e foi realizado um número relativamente reduzido de pontes; 3 ) se, nos casos de insuficiência coronária crônica, o grupo da Escola Paulista de Medicina usa a revascularização miocárdica sem perfusão como um método rotineiro, ou apenas 
VEGA, H.; LEĀO, L. E. V.; FONSECA, J. H. A. P.; GOMES, W. J.; SILVA, L.A. ; BRANCO, J. N. R.; MALUF, M. A.; RIBEIRO, E. E.; BUFFOLO, E. - Revascularização cirúrgica do miocárdio sem utilização do circuito extracorpóreo em pacientes com infarto agudo do miocárdio tratados previamente com estreptoquinase intravenosa. Rev. Bras. Cir. Cardiovasc., 6(3):153$157,1991$.

para aqueles pacientes que apresentam risco na utilização da circulação extracorpórea. Finalmente, achamos que, com a evolução da circulação extracorpórea, cada vez mais segura e com grande diminuição das suas complicaçōes, este método não tem motivos para ser usado rotineiramente. No entanto, cremos que todo cirurgião cardiovascular que trata de insuficiência coronária deve se familiarizar com esta técnica e estar apto a usá-la, pois nos parece de grande valia nos casos em que a perfusão acrescenta um risco excessivo, como, por exemplo, nos pacientes com insuficiência renal em hemodiálise, coagulopatas, com insuficiência pulmonar grave, dissecção da aorta, etc., e, talvez, nos casos aqui apresentados. É mais um arma à nossa disposição, de grande importância. Muito obrigado.

\section{DR. HERMÍNIO VEGA \\ (Encerrando)}

Inicialmente, gostaria de agradecer os comentários do Dr. Antônio Penna. Quanto às suas perguntas: 1) o começo de nossa experiência com revascularização cirúrgica do miocárdio sem CEC, utilizamos o método em pacientes com boa função ventricular e sem outras intercorrências clínicas. Entretanto, com a experiência adquirida no decorrer dos anos, passamos a priorizar os pacientes com intercorrências clínicas graves, tais como renais crônicos, pulmonares, doentes neurológicos e diabéticos; evidentemente, os pacientes com infarto agudo do miocárdio constituem um grupo cujo risco operatório, em relação aos não infartados, é menor. Acreditamos, pois, que, em pacientes portadores de lesōes nas artérias coronárias descendente anterior e/ou coronária direita, a revascularização cirúrgica do miocárdio sem CEC pode ser realizada de maneira rápida e segura, evitando-se, assim, as intercorrências clínicas inerentes à CEC. 2) Embora eu não possa afirmar categoricamente que, se esses pacientes fossem operados com CEC, o resultado teria sido diferente; entretanto, a mortalidade hospitalar de $0 / 25$ nos incentiva a continuar investindo no método. 3) Nos casos de insuficiência coronária crônica e em pacientes que possuem lesões que comprometem a artéria coronária descendente anterior e/ou a artéria coronária direita, utilizamos a revascularizaçāo do miocárdio sem utilização do circuito extracorpóreo de maneira rotineira. Mas uma vez, agradeço os comentários pertinentes do Dr. Penna. 